\title{
The strength-endurance continuum revisited: a critical commentary of the recommendation of different loading ranges for different muscular adaptations
}

\author{
James P. Fisher, James Steele, Patroklos Androulakis-Korakakis, Dave Smith, Paulo Gentil, Jürgen Giessing
}

\begin{abstract}
Objectives: The accepted wisdom within resistance training is that differing loads and corresponding repetition maximum (RM) ranges are optimal for inducing specific adaptations. For example, prominent organizations and their respective publications have typically prescribed heavy loads for maximal strength increases ( $\geq 85 \% 1 \mathrm{RM} / \leq 6 \mathrm{RM})$, more moderate loads for hypertrophy $(67-85 \% 1 \mathrm{RM} / 6-12 \mathrm{RM})$ and lighter loads for local muscular endurance (LME; $\leq 67 \% 1 \mathrm{RM} / \geq 12 \mathrm{RM})$. Since we believe these recommendations originate from a misunderstanding and misinterpretation of DeLorme's strength-endurance continuum, the aim of this narrative review is to discuss the preponderance of research surrounding training load and strength and LME adaptations.
\end{abstract}

Design \& Methods: Narrative Review

Results: The current body of literature fails to support recommendations for the use of specific loads for specific strength, hypertrophy or LME adaptations. Furthermore, that the strength-endurance continuum originally presented by DeLorme was never intended to compare the use of heavier- and lighter-load resistance training, but rather to consider the adaptations to strength training and aerobically based endurance exercise. Finally, a lack of clarity considering absolute- and relative- LME has confounded understanding of this adaptation.

Conclusions: The body of research supports that absolute LME appears to adapt as a result of maximal strength increases. However, relative LME shows minimal response to strength training with either heavier- or lighter-loads. We present the limitations of the current body of research and promote specifically detailed recent research as well as the importance of generality of strength and LME in both sporting and real-world settings.

(Journal of Trainology 2020;9:1-8)

Key words: strength $\boldsymbol{\square}$ local muscular endurance $\boldsymbol{\|}$ load $\boldsymbol{\square}$ intensity $\boldsymbol{\square}$ repetitions, resistance training

\section{INTRODUCTION}

Traditionally, resistance training has been prescribed based upon knowledge of the maximal load a person can lift for a single repetition (i.e., 1-repetition maximum; 1RM) and then training performed using a predetermined percentage of this load based on the desired outcome (e.g. increased strength, hypertrophy, or local muscular endurance; LME). The prominent organisations within strength training (the National Strength and Conditioning Association [NSCA] and American College of Sports Medicine [ACSM], have typically prescribed training loads as follows: $\sim 60-70 \% 1 \mathrm{RM}$ for novice or intermediate trainees, or $\sim 80-100 \% 1 \mathrm{RM}$ for advanced individuals for strength; $\sim 70-85 \% 1 \mathrm{RM}$ for novice or intermediate, or $\sim 70-100 \% 1 \mathrm{RM}$ for advanced individuals for hypertrophy; and $\leq 67 \% 1 \mathrm{RM}$ for local muscular endurance $\left(\mathrm{LME},{ }^{1,2}\right)$. This is often referred to as the repetition maximum continuum or the strength-endurance continuum; the idea that heavier loads optimise strength adaptations and lighter loads optimise LME adaptations. We propose that this is the generally accepted wisdom amongst personal trainers and strength and conditioning coaches based on these long-standing beliefs.

However, current literature ${ }^{3,4}$ supports the view that heavyand light-loads produce equivalent increases in strength (when measured by impartial methods to parse-out improvements in skill due to familiarity of the testing mode) when exercise is continued to momentary failure. For instance, improving maximal strength (as measured by 1RM in a specific exercise) might best be achieved by practicing heavy/ maximal repetitions of that specific exercise (e.g., to improve a bench press 1RM a person might be best advised to practice a bench press $\left.1 \mathrm{RM}^{3-6}\right)$. Certainly, evidence has supported the notion that motor schemata are both highly movement specific as well as load/force specific ${ }^{7}$. As such, this appears dominantly a product of rehearsing the synchronous motor unit recruitment required for maximal lifts (e.g., bench press), as well as their coordinated recruitment patterning for the technical elements of more complex exercises (e.g., clean and jerk). However, evidence suggests that the apparent superiority of heavy/maximal loading might be absent when using impartial testing methods. For example, Mitchell et al. ${ }^{8}$ reported greater increases in $1 \mathrm{RM}$ for groups training with

Received December 16, 2019; accepted January 18, 2020

From the Solent University, East Park Terrace, Southampton, UK (J.P.F., J.S., P.A.K.), Manchester Metropolitan University, Crewe, UK (D.S.),

Faculdade de Educação Física e Dança, Universidade Federal de Goiás, Goiânia, Brazil (P.G.), and University of Koblenz-Landau, Germany (J.G.)

Communicated by Takashi Abe, PhD

Correspondence to: Dr. James P. Fisher, Solent University, East Park Terrace, Southampton, UK.

Email: James.Fisher@solent.ac.uk

Journal of Trainology 2020;9:1-8 @2012 The Active Aging Research Center http://trainology.org/ 
$80 \%$ of $1 \mathrm{RM}$ compared to those training with $30 \% 1 \mathrm{RM}$. However, when tested using isometric torque (an impartial testing method since neither group had trained using isometric contractions) there were no between group differences in strength increases. Fisher, Ironside and Steele ${ }^{9}$ reported similar findings; that dynamic knee extension exercise at either $80 \%$ or $50 \%$ of maximum torque produced similar increases in maximal isometric strength. This is further supported by a recent systematic review and meta-analysis considering high( $>60 \% 1 \mathrm{RM})$ and low- ( $\leq 60 \% 1 \mathrm{RM})$ load training.4 The authors reported statistically significant differences in favour of heavy loads when considering 1RM, yet no significant difference when considering impartial (isometric) strength testing.

Furthermore, evidence suggests that hypertrophic adaptations can be equally attained almost irrespective of training load used..$^{4,8}$ For example, the aforementioned study by Mitchell et al. ${ }^{8}$ reported similar increases in quadriceps muscle volume between groups training at $30 \%$ and $80 \% 1 \mathrm{RM}$. In addition, a comprehensive review comparing loads $>60 \%$ $1 \mathrm{RM}$ to those $\leq 60 \% 1 \mathrm{RM}$ concluded "muscle hypertrophy can be equally achieved across a spectrum of loading rang$e s " 4$. The caveat to these similar increases in muscle size irrespective of load appears to be intensity of effort, that is; that similar adaptations are attained so long as participants train to momentary failure.

In view of these publications it is interesting that there have been no recent reviews of the strength-endurance continuum in consideration of the relationship between maximal strength (e.g., 1RM) and LME adaptations. As such, we believe the area warrants a narrative discussion of the origins of this continuum as well as to discuss often cited articles supposedly supporting a continuum and perhaps attempt to explain why differing adaptations might occur. With this in mind, the aim of this commentary is to discuss the limitations of the notion of the repetition maximum /strength-endurance continuum and provide more evidence-based recommendations for practitioners.

\footnotetext{
Absolute- and Relative- Muscular Endurance

A notable problem with any recommendations pertaining to strength and LME adaptation is the failure to differentiate between absolute and relative LME. Absolute LME should be considered the number of repetitions possible at a given absolute load, whereas relative LME is the number of repetitions possible at a given $\% 1 \mathrm{RM} .{ }^{10}$ In testing, these would be represented by use of either an absolute load (that does not change in relation to strength or following any strength training intervention), or, in contrast, a relative load (that is always measured as a percentage of maximal strength. e.g. \%1RM). The $\mathrm{ACSM}^{2}$ (page 697) stated:

"RT has been shown to increase absolute LME (i.e., the maximal number of repetitions performed with a specific pre-training load), but limited effects are observed in relative LME (i.e., endurance assessed at a specific relative intensity or $\% 1 \mathrm{RM})$...
}

...A relationship exists between increases in strength and LME such that strength training alone may improve endurance to a certain extent."

This statement appears to conflict with the guidance based on loading and repetition ranges. ${ }^{1,2}$ As such we should question why, if absolute LME improves with maximal strength and relative LME shows limited effects, would we recommend that individuals use different training loads or repetition ranges to target strength or LME.

\section{Clarity regarding the work of DeLorme}

The above guidelines by the $\mathrm{ACSM}^{2}$ and $\mathrm{NSCA}^{1}$ cite the work of Anderson and Kearney ${ }^{11}$, Stone and Coulter ${ }^{10}$, and Campos et al. ${ }^{12}$; each of which will be discussed later herein, in support of what has become the accepted "wisdom" in strength and conditioning; the strength-endurance continuum. Each of these studies cites, in turn, the seminal work of DeLorme $^{13}$ where he discusses the use of heavy resistance exercise rather than endurance exercise for restoration of muscular strength and power in injured veterans. However, each of these studies, and many others, appear to have misinterpreted, and as such misrepresented, DeLorme's hypothesis and findings. For example, DeLorme clarifies "By 'powerbuilding' exercises we mean exercises in which a heavy resistance is used for a low number of repetitions. 'Endurancebuilding' exercises are those in which a low-resistance is used for a large number of repetitions" (page 650).

Whilst DeLorme states endurance exercise to be low-resistance/high-repetition, he further clarifies examples as "stairclimbing, walking, bicycling and similar low-resistance exercises" (651). He continues: "How illogical it would be for a track man to train for long-distance running events solely by doing knee bends with heavy weights on his shoulder, or a professional weight-lifter to train for heavy lifts solely by running several miles a day" (page 651). In fact, within his 1945 article he provides guidance that "the workout must begin with a weight considerably less than the 10RM, so that when the 10RM has been reached, seventy to 100 repetitions have been performed" (page 648). Notably this number of repetitions was completed across 7-10 sets of 10 repetitions. Furthermore, DeLorme does not use the term muscular endurance throughout this article, in contrast to what other authors have stated $^{10-12}$. Instead, DeLorme was making reference to what is now considered aerobically based endurance exercise modalities (e.g., "stairclimbing, walking, bicycling" and "running several miles a day"). In this discussion of the work of DeLorme it is important to clarify that he developed these ideas in later publications, and in 1948 DeLorme and Watkins ${ }^{14}$ clarified that "it has become apparent that the term 'heavy resistance exercises' bears false implications, and the term 'progressive resistance exercises' was suggested as being far more appropriate" (page 263). It is, thus, evident that DeLorme did not intend to differentiate between heavy- and light-load resistance training per se, but rather to determine the disparity in adaptations between progressive resistance exercise and aerobically based endurance exercise. It is per- 
haps also noteworthy that in this article DeLorme and Watkins ${ }^{14}$ suggested a decreased training volume, from 7-10 sets to, the now commonly accepted, 3 sets of 10 repetitions. Perhaps notably though, this was described in the following format;

First set of 10 repetitions - use $1 / 2$ of 10 repetition maximum Second set of 10 repetitions - use $3 / 4$ of 10 repetition maximum

Third set of 10 repetitions - use 10 repetition maximum

In this case DeLorme appears to be suggesting 2 submaximal sets and then a single set to repetition maximum, with the technical advice that:

"The movements are done smoothly, rhythmically, and without haste, but not so slowly that the mere holding of the weight will tire the patient. Quick or sudden motions while exercising are to be avoided." (page 646).

It is unclear as to whether DeLorme was suggesting to train to momentary failure, or to a repetition maximum, as have been recently more clearly defined.$^{15}$ Nevertheless, it is clear that the final set in this protocol was intended to require at least a near maximal effort. It is perhaps also worth mentioning that DeLorme ${ }^{13}$ (page 649) describes the principles of double-progression; that, when performing a given exercise, as the number of repetitions increases beyond a target range (e.g., 8-12) a person should increase the load being used, which has the subsequent effect of reducing the repetitions possible, and so the trainee repeats the process as he or she becomes stronger. Whilst DeLorme does not provide a citation for this concept our research suggests that this originates from Allan Calvert in his text "The First Course in BodyBuilding and Muscle Developing Exercises". ${ }^{16}$ As an example of double progression; a person who could initially only perform 8 repetitions with a load of $100 \mathrm{~kg}$ progresses over time to be able to perform 12 repetitions with $100 \mathrm{~kg}$ (with all other exercise factors being equal; repetition duration, range of motion, exercise technique, etc.). He or she then increases the load to $105 \mathrm{~kg}$ and the number of repetitions which can be performed decreases to 8 repetitions. If we accept that an 8RM is a measure of strength, then the necessity of this principle to increase strength is something of a paradox; it is built on a premise that as we increase the number of repetitions we perform we increase strength sufficiently to increase the training load. But that increasing the load is important to continue increasing strength. Ultimately, this principle is anecdotally used to support the strength-endurance continuum and that of specificity: lifting heavier weights increases maximal strength, whilst performing a greater number of repetitions with a lighter weight increases local muscular endurance. However, it seems far more likely that double progression in this sense represents a more time-efficient, but not essential, method of training. For example, if performed at a repetition duration of 2 s concentric: $2 \mathrm{~s}$ eccentric, performing 8-12 repetitions represents a time of 32-48 seconds. If a person were to continue increasing strength with an absolute load, and thus continue increasing the number of repetitions they could perform, then this time under load might become impractical, and other sensations such as discomfort might become a factor for exercise cessation (see later section on effort and discomfort)

\section{Repetition Maximum Continuum}

Within the fourth edition of "Essentials of Strength Training and Conditioning", Haff and Triplett ${ }^{1}$ provide a table (17.9, page 458) clarifying loads/repetition ranges for optimizing specific strength $(\geq 85 \% 1 \mathrm{RM} / \leq 6 \mathrm{RM})$, hypertrophy $(67-85 \%$ $1 \mathrm{RM} / 6-12 \mathrm{RM})$, and muscular endurance $(\leq 67 \% 1 \mathrm{RM} / \geq$ 12RM) adaptations. However, the references cited do not wholly support these recommendations. For example, two of the eight citations are textbooks $s^{17,18}$, and a third citation is a chapter from a book. ${ }^{19}$ The fourth and fifth citations are a commentary/review article ${ }^{20}$, and an article aimed at providing strength training recommendations by Kraemer and Koziris $^{21}$. None of these citations are empirical studies presenting data. Of the remaining three citations, the first empirical study, that of Berger ${ }^{22}$, considered bench press strength increases for 199 male college students following a 12-week intervention. Berger ${ }^{22}$ reported more favorable strength increases for 8RM training compared to both higher- (10RM and $12 \mathrm{RM}$ ) and lower- (2RM) repetition maximum training. The second empirical study, that of Herrick and Stone ${ }^{23}$, compared previously untrained females divided into one of two groups; either progressive resistance exercise (PRE; 3 sets of $6 \mathrm{RM}$ for 15 weeks) or periodized resistance exercise (PER; 8 weeks of 3 sets using 10RM, 2 weeks of 3 sets using 4RM and 2 weeks of 3 sets using 2RM, with 1 week of active rest between each cycle). The authors measured bench press and back squat pre- and post-intervention as well as every 3 weeks (totaling 6 testing time points) finding significant within-group strength increases for both groups with no betweengroups differences. The third empirical study, by Tesch and Larsson $^{24}$, took biopsies from the vastus lateralis and medial deltoid muscles from 3 competitive bodybuilders, as well as measuring strength of the quadriceps using isokinetic dynamometry. They compared this data to reference groups of physical education students and national elite power- and weight-lifters. They did not conduct an intervention that compared training adaptations following the proposed differing repetition ranges. Interestingly, the authors reported similar muscle morphology between the bodybuilders and the physical education students ( $\%$ fast twitch muscle fibres, $\%$ fast twitch muscle area, fast twitch: slow twitch ratio, as well as fast twitch-, slow twitch- and mean- fibre area), stating:

"We did not observe any sign of individual muscle fiber enlargement in either thigh or shoulder muscles of successful bodybuilders. Thus, despite the considerably greater body weight per height and less body fat in bodybuilders compared to habitually trained and age matched men, mean fiber area did not differ." (page 305).

It can only be assumed that this study was cited based on the comments in the discussion that bodybuilders typically perform 3 or more sets of 6-12 repetitions to concentric fail- 
ure, interspersed with short recovery periods - which is aligned to the NSCA recommendation cited above. ${ }^{1}$ However, based on this as well as the previous citations it is not clear how Haff and Triplett ${ }^{1}$, nor the organization (NSCA), are able to justifiably endorse such recommendations.

\section{Support for a Repetition-Maximum Continuum}

Since we have clarified that the strength-endurance continuum was never proposed to present disparate training adaptations to heavier- and lighter-loads in resistance training, we shall use the term repetition-maximum continuum in further discussion of this concept. The three often cited empirical studies used (by the ACSM/NSCA) to support the repetition maximum continuum shall now be considered chronologically.

\section{Anderson and Kearney ${ }^{11}$}

The publication by Anderson and Kearney ${ }^{11}$ is a common citation in favor of the repetition maximum continuum and as such, it is worth discussing in detail the research design. Forty-three untrained males were divided into heavy-load (HL, $\mathrm{n}=15$; 3sets of $6-8 \mathrm{RM}$ ), moderate load (ML, $\mathrm{n}=16 ; 2$ sets of 30-40RM), and light-load (LL, $n=12$; 1 set of 100150RM) training groups. Participants trained using the bench press exercise $3 \mathrm{x} /$ week for 9 weeks. The authors reported pre- and post-intervention 1RM, repetitions for absolute-LME using $27.23 \mathrm{~kg}$, and repetitions for relative-LME using $40 \%$ of pre- and post-intervention 1RM. Analysis of variance revealed significant increases in $1 \mathrm{RM}$ for all groups $(\mathrm{HL}=13.7 \mathrm{~kg}, \mathrm{ML}=5.4 \mathrm{~kg}$, and $\mathrm{LL}=3.2 \mathrm{~kg})$ with a significant group $\mathrm{x}$ time interaction. The authors found significant pre- to post-intervention increases for absolute-LME $(\mathrm{p}<0.0001$; $\mathrm{HL}=9.5, \mathrm{ML}=14.4$, and $\mathrm{LL}=14.6$ repetitions), but betweengroup differences were not statistically significant $(p<0.13)$. For relative-LME the authors reported significant pre- to post-intervention changes $(p<0.0001)$ as well as group $x$ test interaction $(p<0.0001)$. Follow up analyses revealed significant increases in the number of repetitions for ML and LL groups which were also statistically significantly greater than the change in repetitions for the HL group. There were no significant differences for the change in the repetitions between the ML and LL groups. The values for change in repetitions from pre- to post-intervention for relative-LME were $\mathrm{HL}=$ -2.86 repetitions $/-6.99 \%, M L=8.81$ repetitions $/+22.45 \%$, and $\mathrm{LL}=10.67$ repetitions $/+28.45 \%$. The authors reported a tempo of 40 repetitions per minute, for muscular endurance testing as well as training for the ML and LL groups.

It is interesting that whilst the HL group increased their $1 \mathrm{RM}$ by $13.7 \mathrm{~kg}$ (compared to only $5.4 \mathrm{~kg}$ and $3.2 \mathrm{~kg}$ for ML and LL groups, respectively), the number of repetitions they were able to perform at the absolute load of $27.23 \mathrm{~kg}$ (equivalent to $40 \%$ of their pre-intervention $1 \mathrm{RM}$, and only $33 \%$ of their post-intervention 1RM) increased to a lesser degree (although not significantly so) than the ML and LL groups (14.4 and 14.6 repetitions, respectively). Furthermore, that when performing repetitions assessed for relative-LME at $40 \% 1 \mathrm{RM}$, the ML and LL groups achieved increases in the number of repetitions with an increased load (8.8 and 10.7 repetitions, respectively). In considering the data in more detail and by group, the LL group increased their 1RM by 3.2 $\mathrm{kg}$, which resulted in an increase to their load at relative-LME $(40 \%)$ of only $1.3 \mathrm{~kg}$, whilst the ML group increased their $1 \mathrm{RM}$ by $5.4 \mathrm{~kg}$, which resulted in an increase to their load at relative-LME (40\%) of only $2 \mathrm{~kg}$. It seems reasonable to suggest that the increased number of repetitions for relative-LME for the ML and LL groups might be a result of such small changes in the loads used (i.e. $<2 \mathrm{~kg}$ ). In this sense, the relative-LME test might have been closer to an absolute-LME test.

It is interesting that, in the introduction, Anderson and Kearney ${ }^{11}$ rationalize their own research by citing three studies $^{25-27}$ which they claim challenge the observations of DeLorme ${ }^{13}$. However, these studies, as well as their own, compare heavy-load, low-repetition vs. light load, high-repetition resistance training. As clarified, this study design does not resemble, and thus does not challenge, DeLorme's observations regarding progressive resistance exercise compared with aerobically based endurance modalities. Furthermore, each of these three studies supported that both heavy- and light-loads produce similar enhancement of muscular strength and LME. Indeed, Anderson and Kearney ${ }^{11}$ stated that the findings of Delateur et al. ${ }^{26}$ revealed that "choice of weights is not of prime importance as long as the repetitions are continued to the point of fatigue" (page 248). They continue "Strength and endurance thus appear to be two closely related attributes of the well-trained muscle" (page 248).

Stone and Coulter ${ }^{10}$

A further study considering heavy-, moderate- and lighterloads in relation to increases in 1RM, absolute-LME and relative-LME is that of Stone and Coulter ${ }^{10}$. The authors divided fifty untrained females into heavy-load $(\mathrm{HL}, \mathrm{n}=17$; 3 sets of 6-8RM), moderate load (ML, $\mathrm{n}=16$; 2 sets of $15-20 \mathrm{RM}$ ), and light-load ( $\mathrm{LL}, \mathrm{n}=17$; 1 set of $30-40 \mathrm{RM}$ ) training groups. Testing was performed for bench press (BP) and back squat (BS) exercises with maximal strength measured using 1RM, absolute-LME measured as repetitions at $15.9 \mathrm{~kg}$ (BP) and $25 \mathrm{~kg}$ (BS), and relative-LME measured using repetitions at 45\%1RM (BP) and 55\% 1RM (BS). The authors stated that relative-LME was tested at two loads; load 1 involved preand post-testing repetitions at a given percentage of the preintervention 1RM. However, re-testing muscular endurance post intervention using the same pre-intervention $\% 1 \mathrm{RM}$ is absolute- not relative-LME. Thus, the authors performed two absolute-LME tests; for bench press they used $15.9 \mathrm{~kg}$ and $45 \%$ of pre-intervention 1RM, and for back squat they used $25 \mathrm{~kg}$ and $55 \%$ of pre-intervention $1 \mathrm{RM}$. The second relativeLME test using load 2 was a true relative-LME test where the pre-test used a $\%$ of the pre-intervention 1RM, and the posttest used the same percentage of the post-intervention 1RM. Tempo was described as 40 and 30 repetitions per minute for the bench press and back squat, respectively. The authors reported no significant differences between the three protocols for improvements in 1RM, or absolute-LME, and reported no significant increases in relative-LME. It is, therefore, surprising that the $\mathrm{NSCA}^{1} / \mathrm{ACSM}^{2}$ cited this paper for the use 
of higher repetition ranges for improving muscular endurance when the results do not support this claim.

\section{Campos et al. ${ }^{12}$}

Perhaps the most notable research cited to support a repetition-maximum continuum is that of Campos et al. ${ }^{12}$. Thirtytwo physically active but previously untrained young males were divided into low repetition (Low Rep; 3-5RM, $n=9$ ), intermediate repetition (Int Rep; 9-11RM, $\mathrm{n}=11$ ), high repetition (High Rep; 20-28RM, n=7), and non-exercising control $(\mathrm{CON} ; \mathrm{n}=5)$ groups. The exercising groups performed leg press, squat and knee extension exercises $2 \mathrm{x}$ /week for the first 4 weeks and $3 x /$ week for the last 4 weeks. Participants were tested pre- and post-intervention for 1RM and, after a 4-5 minute recovery, they completed as many repetitions as possible with $60 \%$ 1RM (the authors do not clarify whether this was relative- or absolute- LME in the article, but this description and personal correspondence have confirmed this to be a test of relative LME). The Low Rep group showed significantly greater 1RM increases in leg press and squat exercises compared to the other groups, but the increase in knee extension 1RM was significantly greater than the High Rep group only. The authors reported that the three training groups significantly improved in the number of repetitions performed with $60 \% 1 \mathrm{RM}$ in the squat exercise, but neither the Int Rep group nor the Low Rep group showed a significant improvement in the leg press or knee extension exercises. In fact, the number of repetitions significantly decreased in the Low Rep group for the leg press. The authors did not report a tempo or repetition duration for testing or training.

The authors provide data in the form of figures, rather than specific numerical values, and as such these cannot be discussed in detail. Indeed, in private correspondence we have been advised that the raw data is no longer available for consideration/analyses. However, in an attempt to better consider this study, we have used a digitization program to calculate the values from Figure 3 in the $\operatorname{article}^{12}$ (WebPlotDigitizer, v3.12; Ankit Rohgati; http://arohatgi.info/WebPlotDigitizer/ index.html). We estimate pre-intervention $1 \mathrm{RM}$ values for the leg press to be $\sim 310 \mathrm{~kg}$ for Low Rep, $\sim 294 \mathrm{~kg}$ for Int Rep, and $\sim 300 \mathrm{~kg}$ for High Rep (reported as not significantly different between groups). The post-intervention 1RM values are estimated as $\sim 498 \mathrm{~kg}$ for Low Rep (reported as $61 \%$ improvement), 398kg for Int Rep (reported as 36\% improvement) and $\sim 364 \mathrm{~kg}$ for High Rep (reported as 32\% improvement, although using the values extracted using the digitization software we calculate a $21 \%$ improvement). With a similar method using the authors' Figure 4, the local muscular endurance testing appears to have produced estimated pre-intervention values for the leg press of $\sim 40$ repetitions for Low Rep, $\sim 39$ repetition for Int Rep, and $\sim 35$ repetitions for High Rep. The corresponding estimated post-testing repetitions were $\sim 32$ repetitions for Low Rep (reported as -20\% change), 43 repetitions for Int Rep (reported as 10\% improvement), and 68 repetitions for High Rep (reported as 94\% improvement).

It is, therefore, surprising that the low rep group, increasing their $1 \mathrm{RM}$ strength by $\sim 188 \mathrm{~kg} / 61 \%$ decreased their relative muscular endurance from 40 repetitions with $\sim 186 \mathrm{~kg}$ to 32 repetitions with $\sim 298 \mathrm{~kg}$. In contrast, and more surprisingly, the high rep group increased their 1RM strength by $\sim 64 \mathrm{~kg} / 21 \%$ but also increased their relative muscular endurance from 35 repetitions with $\sim 180 \mathrm{~kg}$ to 68 repetitions with $\sim 218 \mathrm{~kg}$. Aside from the possibility that low rep training truly does produce greater maximal strength increases but diminish relative muscular endurance performance whilst high rep training produces (albeit lesser) strength increases and far superior relative muscular endurance improvements (compared to low rep training), we have hypothesized other factors which might have confounded these results. For example (and while we believe this to be highly improbable), though participants were randomized the relatively small sample size per group may have resulted in heterogeneous groupings regarding genetic predisposition to either maximal strength increases or muscular endurance increases for low- and high-rep groups respectively. Indeed, the wide heterogeneity in response variation to resistance training is well known ${ }^{28}$ and, though it is difficult to differentiate true intervention response variation from other random variation ${ }^{29}$, it is well evidenced that some individuals can show considerable improvements in some outcomes but not others and vice versa $^{30,31}$. Another factor, might be that the strength increase for the low rep group could be partially a result of improved motor schema in the practice of synchronous recruitment ${ }^{3}$, and the skill of the exercise itself particularly as the testing resembled the training (although we might expect lower skill increases in a leg press exercise, whether plate loaded or selectorized compared to a more complex movement such as a squat). Finally, and as discussed later, we might consider that the high rep group became acclimatized to the discomfort of performing a greater number of repetitions than the low rep group (20-28RM compared to 3-5RM, respectively) throughout the 8 -week training intervention.

Interestingly despite each of these studies being used to support a repetition maximum continuum of $\leq 6 \mathrm{RM}$ for strength, 6-12RM for hypertrophy, and $\geq 12 \mathrm{RM}$ for LME adaptations ( $\mathrm{NSCA}^{1}$, page 458), only the study by Campos et al. ${ }^{12}$ study tested these repetition ranges (e.g. strength [low rep] 3-5RM, hypertrophy [int rep] 9-11RM, and LME [high rep] 20-28RM). Furthermore, only Campos et al. ${ }^{12}$ took any measurement of muscle hypertrophy. The authors reported that a hypertrophic effect was observed in type I, IIA and IIB muscle fibre types in the low- and intermediate-RM training groups only. There were no significant differences between the low- and intermediate- RM groups for muscle fibre hypertrophy (low $\mathrm{RM}=12.4,22.9,25.3 \%$ and intermediate $\mathrm{RM}=13.1,16.3,27.2 \%$, for type I, IIA, and IIB fibre types, respectively).

\section{Effort and Discomfort}

When we consider heavier- and lighter-load RT, a factor that might become important when trying to exercise to momentary failure is that of discomfort compared to effort. There is a growing body of research supporting that discomfort (defined as the physiological and unpleasant sensations associated with exercise $\mathrm{e}^{32,33}$ ) is greater when performing exer- 
cise to momentary failure using a lighter- $(50 \% \mathrm{MVC})$ compared to a heavier- (80\% MVC) load in both males and females $^{9,34}$. This is potentially a result of increased blood lactate and cortisol accumulation ${ }^{35}$ and primarily thought to result from afferent feedback mechanisms ${ }^{33}$. As such, it seems likely that the ability to reach momentary failure with lighter loads could be impaired by the discomfort during resistance exercise $^{36}$. This, in turn, might negatively impact the chronic muscular adaptations across the duration of an intervention when compared to a heavier-load group, who can reach momentary failure and attain the desired effort with far less discomfort. An example might be the Anderson and Kearney ${ }^{11}$ study where participants in the HL group performed 3 sets of 6-8 repetitions to momentary failure, whereas the ML and LL group performed 30-40 and 100-150 repetitions respectively. At the rate of 40 repetitions per minute this equates to $\leq 12$ seconds per set for the HL group (performing 3 sets $=36$ seconds under load), 60 seconds per set for the ML group (performing 2 sets $=2$ minutes under load) and 3 minutes 40 seconds per set for the LL group.

Furthermore, the changes to LME testing for lighter-load/ higher repetition groups might be a potential adaptation to discomfort as a result of the training intervention, i.e., performing very high-repetition sets; e.g. 20-28RM ${ }^{12}, 30-40^{10,11}$ and $100-150 \mathrm{RM}^{11}$. A test of LME will likely stimulate considerable discomfort as a person nears maximal effort and task failure and as such training at lighter loads/higher repetitions might not incur neuromuscular or morphological adaptations that improve LME but rather accommodate familiarisation and increased tolerance of the discomfort associated with a test of LME. Certainly, recent work has shown that repeated exposure to exercise conditions known to cause discomfort, such as high intensity interval training, increases pain tolerance and this might partly explain improvements in time to task failure ${ }^{37}$. Indeed, there might be mechanisms beyond muscular adaptations by which the repetition maximum continuum applies. For example, by training at heavier/maximal loads a person might improve strength more than at lighter loads as a result of enhanced skill, along with practicing high synchronous motor unit recruitment, and by training at lighter loads a person might improve LME as a result of the familiarisation and tolerance to the discomfort of lighter-load exercise.

\section{Hypertrophic adaptations}

Whilst this narrative review has primarily been focused upon the concurrent adaptations in maximal strength and absolute muscular endurance across a range of training loads, hypertrophy has often been considered in context of the few respective studies which have tested the "hypertrophy-zone" of the repetition maximum continuum (e.g. 67-85\% 1RM/6$12 \mathrm{RM}^{1}$ ). With a dearth of research, both the $\mathrm{NSCA}^{1}$ and $\mathrm{ACSM}^{2}$ have failed to provided support for the claims that this loading range is optimal for muscular growth. As discussed earlier, the repetition maximum continuum, displayed as table 17.9 (page 458) in "Essentials of Strength Training and Conditioning" cites only 3 empirical studies. Those of
Berger $^{22}$ and Herrick and Stone ${ }^{23}$ did not take any measurement of muscle size. The final study, that of Tesch and Larsson $^{24}$, was observational in comparing intramuscular properties following biopsy between elite bodybuilders and reference groups of physical education students. The authors did not perform pre- or post-intervention measurements and did not consider different loads/repetition ranges to scientifically test the repetition maximum continuum. More so, whilst the authors suggest that bodybuilders typically train using 3 sets of 6-12 repetitions, the data presented does not support that this is optimal for muscle growth. In fact, as stated above, the authors report similar muscle morphology when comparing the elite bodybuilders to the physical education reference group.

Of the other often cited studies, neither Anderson and Kearney ${ }^{11}$, nor Stone and Coulter ${ }^{10}$ took measurements of muscle size. Campos et al. ${ }^{12}$ considered hypertrophic adaptations measured by muscle biopsy in assessment of the repetition maximum continuum, reporting similar increases in type I, IIA and IIB muscle fibres between the groups which trained using low reps $(3-5 \mathrm{RM})$ and intermediate reps (9-11RM).

More recent publications provide conflicting evidence to a repetition maximum continuum. Mitchell et al. ${ }^{8}$ reported similar increases in quadriceps muscle volume between groups training using $30 \%$ and $80 \% 1 \mathrm{RM}$, and a meta-analysis and systematic review supported that hypertrophic adaptations are similar between loads $>60 \% 1 \mathrm{RM}$ and loads $<60 \%$ $1 \mathrm{RM} \mathrm{M}^{4}$.

\section{CONCLUSIONS}

The aim of this piece was to review and challenge the commonly accepted wisdom of the repetition-maximum continuum as well as to clarify the work of Thomas DeLorme and the strength-endurance continuum. The data and discussions presented suggest that the guidance of specific loads/repetition ranges for strength, hypertrophy, or LME are not supported by the evidence. When reviewing the data it appears that strength increases are not only attainable, but effectively the same, at both very heavy (maximal/near maximal) loads and more moderate loads of $8-12 \mathrm{RM}$. Furthermore, that as strength increases so too does absolute-LME. Finally, that hypertrophic adaptations appear similar across a spectrum of loading ranges where exercise is performed to momentary failure.

Whilst many persons train for maximal strength, and as such might practice heavy/maximal resistance training under the guidance of "practicing the test", we should not undervalue the importance of adaptations in absolute LME in both sports and real-world activities. The reality is that a person seldom performs single maximal efforts, but rather repeated muscle actions. A prime example in a sporting environment might be the $225 \mathrm{lb}$ bench press for as many repetitions as possible in the NFL Combine test. Prospective athletes are not asked to perform a 1RM test but rather a test of absolute LME (e.g. the load is set at $225 \mathrm{lbs}$ irrespective of player position or weight). A lay example might be the movement of our own 
bodyweight, which, whilst it might fluctuate to a degree, is generally relatively constant; as such tests of press-ups, pullups, or dips, as well as more functional tasks such as rising from a chair, climbing a flight of stairs, etc. are effectively tests of absolute LME.

\section{Practical Applications}

With the above in mind, and since training specifically for strength, hypertrophy or LME is often performed as part of a periodised resistance training programme we caution strength and conditioning coaches, personal trainers and trainees in the use of specific loads/repetition ranges for specific outcomes (e.g. strength, hypertrophy, LME). It is our view that the preponderance of research does not support a repetitionmaximum continuum, but rather we suggest that resistance training be performed to a high degree of effort and that loads are self-selected based on convenience, safety, and psychophysiological factors such as discomfort, etc. The data suggests that following this guidance similar strength adaptations will occur irrespective of load, and that absolute LME increases with maximal strength, whereas relative LME shows little response to strength increases. Persons training specifically for muscle hypertrophy are able to self-select a load based on personal preference, accessibility, etc.

\section{Compliance with ethical standards \\ Funding}

No sources of funding were used to assist in the preparation of this article.

\section{Conflicts of Interest}

James Fisher, James Steele, Patroklos AndroulakisKorakakis, Dave Smith, Jürgen Giessing, and Paulo Gentil declare that they have no conflicts of interest relevant to the content of this article.

\section{REFERENCES}

1. Haff GG, Triplett NT. Essentials of Strength Training and Conditioning, 4th Ed. Champaign, Human Kinetics, 2016.

2. Ratamess NA, Alvar BA, Evetoch TK, et al. Progression models in resistance training for healthy adults. Med Sci Sports Exerc 2009;41:687708.

3. Fisher J, Steele J, Smith D. High- and Low-Load Resistance Training: Interpretation and Practical Application of Current Research Findings. Sport Med 2017;47:393-400.

4. Schoenfeld BJ, Grgic J, Ogborn D, et al. Strength and hypertrophy adaptations between low- versus high-load resistance training: A Systematic review and meta-analysis. J Strength Cond Res 2017;31:35083523 .

5. Mattocks KT, Buckner AL, Jessee MB, et al. Practicing the test produces strength equivalent to higher volume training. Med Sci Sports Exerc 2017; 49:1945-1954

6. Dankel SJ, Counts BR, Barnett BE, et al. Muscle adaptations following 21 consecutive days of strength test familiarization compared with traditional training. Muscle Nerve 2017;56:307-314.

7. Schmidt RA. Motor schema theory after 27 years: reflections and implications for a new theory. Res Q Exerc Sport 2003; 74:366-75.

8. Mitchell CJ, Churchward-Venne TA, West DWD, et al. Resistance exercise load does not determine training- mediated hypertrophic gains in young men. J Appl Physiol 2012;113:71-77.

9. Fisher J, Ironside M, Steele J. Heavier- and lighter-load resistance training to momentary failure produce similar increases in strength with differing degrees of discomfort. Muscle Nerve 2017;56:797-803.

10. Stone WJ, Coulter SP. Strength/endurance effects from three resistance training protocols with women. J Strength Cond Res 1994;8:231-234.

11. Anderson T, Kearney JT. Effects of three resistance training programs on muscular strength and absolute and relative endurance. Res $Q$ 1982;53:1-7.

12. Campos GER, Luecke TJ, Wendeln HK, et al. Muscular adaptations in response to three different resistance-training regimens: specificity of repetition maximum training zones. Eur J Appl Physiol 2002;88:50-60.

13. DeLorme TL. Restoration of muscle power by heavy resistance exercise. $J$ Bone Joint Surg 1945;27:645-667.

14. DeLorme TL, Watkins AL. Technics of Progressive Resistance Exercise Arch Phys Med Rehab 1948;29:263-273.

15. Steele J, Fisher J, Giessing J, et al. Clarity in reporting terminology and definitions of set endpoints in resistance training. Muscle Nerve 2017;56: 368-374.

16. Calvert A. The First Course in BodyBuilding and Muscle Developing Exercises. Philadelphia, The Milo Barbell System, 1911.

17. Fleck SJ, Kraemer WJ. Designing Resistance Training Programs. 4th Ed. Champaign, Human Kinetics, 2014

18. Stone $\mathrm{MH}, \mathrm{O}$ 'Bryant HS. Weight training: A scientific approach. Minneapolis, Burgess, 1987.

19. Tesch PA. Training for Bodybuilding. In The encyclopaedia of Sports Medicine: Strength and Power in Sport. 1st Ed. Komi PV, ed Malden MA. Blackwell Scientific, 1992.

20. Hedrick A. Training for hypertrophy. Strength Cond J 1995;17:22-29.

21. Kraemer WJ, Koziris LP. Muscle strength training; techniques and considerations. Phys Ther Pract 1992;2:54-68.

22. Berger RA, Optimum repetitions for the development of strength. Res $Q$ 1962;33:168-181.

23. Herrick AR, Stone MH. The effects of periodization versus progressive resistance exercise on upper and lower body strength in women. $J$ Strength Cond Res 1996;10:72-76.

24. Tesch PA, Larson L. Muscle hypertrophy in bodybuilders. Eur J Appl Physiol 1982;49:301-306.

25. Clarke DH, Stull GA. Endurance training as a determinant of strength and fatigability. Res $Q$ 1970;41:19-26.

26. DeLateur BJ, Lehmann JF, Fordyce WE. A test of the DeLorme axiom. Arch Phys Med Rehab 1968;49:245-248

27. Stull GA, Clarke DH. High-resistance, low repetition training as a determiner of strength and fatigability. Res $Q$ 1970;41:189-193.

28. Hubal MJ, Gordish-Dressman H, Thompson PD, et al. Variability in muscle size and strength gain after unilateral resistance training. Med Sci Sports Exerc 2005;37:964-972.

29. Atkinson G, Williamson P, Batterham AM. Issues in the determination of 'responders' and 'non-responders' in physiological research. Exp Physiol 2019;104:1215-1225.

30. Churchward-Venne TA, Tieland M, Verdicj LB, et al. There Are No Nonresponders to Resistance-Type Exercise Training in Older Men and Women. J Am Med Dir Assoc 2015; 16:400-411.

31. Barbalho MSM, Gentil P, Izquierdo M, et al. There are no no-responders to low or high resistance training volumes among older women. Exp Gerontol 2017;99:18-26.

32. Abbiss CR, Peiffer JJ, Meeusen R, et al. Role of ratings of perceived exertion during self-paced exercise: what are we actually measuring? Sports Med 2015;45:1235-1243.

33. Marcora S. Perception of effort during exercise is independent of afferent feedback from skeletal muscles, heart, and lungs. J Appl Physiol 2009;106: 2060-2062.

34. Stuart C, Steele J, Gentil P, et al. Fatigue and perceptual responses of heavier- and lighter-load isolated lumbar extension resistance exercise in males and females. PeerJ 2018;6:e4523.

35. Genner, KM, Weston, M. A comparison of workload quantification methods in relation to physiological responses to resistance exercise. $J$ Strength Cond Res 2014;28:2621-2627. 
36. Staiano W, Bosio A, de Morree HM, et al. The cardinal exercise stopper: Muscle fatigue, muscle pain or perception of effort? Prog Brain Res 2018; 240:175-200.
37. O'Leary TJ, Collett J, Howells K, et al. High but not moderate-intensity endurance training increases pain tolerance: a randomised trial. Eur J Appl Physiol 2017;117:2201-2210. 